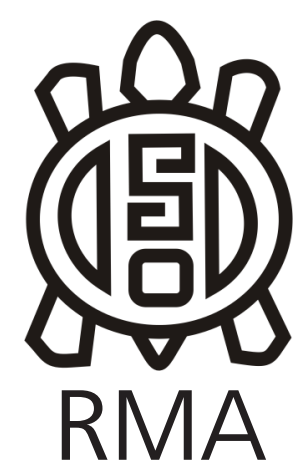

Arqueología

\title{
Más allá de la Puna: Artefactos "Saladillo" del Valle Calchaquí (Cachi, Salta, Argentina) como evidencia de ocupaciones humanas del Holoceno Medio
}

\author{
Beyond the Puna: "Saladillo" Artifacts from the Calchaquí Valley (Cachi, \\ Salta, Argentina) as an evidence of Middle Holocene human occupations \\ Federico Restifo
}

CONICET-Instituto de Investigaciones en Ciencias Sociales y Humanidades (ICSOH). Facultad de Humanidades, Universidad Nacional de Salta, Argentina.

E-mail: federicorestifo@gmail.com

\begin{abstract}
Resumen
Se presenta el análisis de atributos cualitativos y cuantitativos de un conjunto de artefactos Saladillo recuperado en el sector Norte del valle Calchaquí, el cual se encuentra disponible en el Museo Arqueológico de Cachi, provincia de Salta (Argentina) (2500 msnm). El análisis indica similitudes notorias entre el conjunto analizado y aquellos procedentes de la puna de Jujuy y Salta (>3000 msnm). Esto conduce a pensar en la presencia de ocupaciones humanas correspondientes al segmento de finales del Holoceno medio en el sector norte del valle Calchaquí, sobre la base de los fechados absolutos obtenidos en la puna para esta clase de artefacto. A su vez, partiendo de esta idea, pueden sugerirse procesos culturales que habrían posibilitado la distribución de estos artefactos tanto en puna como en valles, considerando desplazamientos de grupos humanos así como transmisión de información. Estas ideas constituyen un punto de partida para guiar la investigación sobre poblaciones humanas anteriores a los ca. 3000 AP en sectores altitudinales más allá de la puna. Se destaca la relevancia de pensar temáticas de investigación en materia de "cazadores recolectores" que integren el amplio espacio del Noroeste Argentino.
\end{abstract}

Palabras clave: Cazadores recolectores; Puna; Valle Calchaquí; Artefactos líticos; Holoceno medio

\begin{abstract}
The analysis of cualitative and cuantitative attributes of an assemblage of "Saladillo" artifact from northern Calchaqui valley is presented. The assemblage is available at the Archaeological Museum of Cachi, Salta Province (Argentina) (2500 msnm). The analysis shows similar attributes between the analized assemblage and those from de puna region (>3000 msnm). These results allow to think about end mid Holocene human occupations in the northern Calchaqui valley, taking into account the absolute dates from puna region artifacts. Hence, considering this idea as an starting point, cultural processes that could explain the distribution of this artifact class in valleys and puna, as mobility and information transmission, are proposed. These ideas are thinked as a guide for the research on the human populations before the ca. 3000 AP in ecological zones lower than puna. The relevance of think about research topics on "hunter gatherers" that allows the integration of results of the wide space of the Argentinean Northwest is highlighted.
\end{abstract}

Keywords: Hunter Gatherers; Puna; Calchaquí Valley; Lithic Artifacts; Middle Holocene

En el Noroeste Argentino (NOA de aquí en adelante), las investigaciones centradas en cronologías anteriores a los 3000 años AP se desarrollaron principalmente en base a los trabajos realizados en tierras altas, por encima de los 3000-3500 msnm, correspondientes a la puna y quebradas de altura (Boman [1908] 1991, Hernández Llosas 2001, Martínez et al. 2013, entre otros). Esto pudo deberse, al menos en parte, a la escasez de hallazgos de sitios estratificados para tal cronología en las tierras de menor elevación, correspondientes a los valles mesotermales y selvas o yungas, en las que la investigación se focalizó en cronologías posteriores a los 3000 años AP mencionados. Sin embargo, tal escasez de hallazgos no necesariamente sería el reflejo directo de la intensidad de ocupaciones tempranas en los sectores de menor altitud que la puna. En este sentido, se destaca la identificación de sitios de superficie con significativas densidades de artefactos como la cantera taller Ampajango, (Cigliano et al. 1962, Carbonelli 2013) y los hallazgos de puntas de proyectil en superficie en el valle de Santa María, que comparadas con las halladas en estratigrafía en la puna podrían indicar una cronología relativa temprana (Agnolin y Carbonelli 2017, Restifo et al. 2018).

Los casos señalados corresponden al sector de valles de la provincia de Catamarca. A su vez, podría sumarse 
el caso de Campo Blanco, en Amaicha del Valle (2000 msnm), Tucumán, con presencia de puntas de proyectil en superficie también asignables a cronologías tempranas, según la secuencia de sitios puneños con fechados absolutos (Hocsman et al. 2003). Particularmente, para el caso del sector Norte del valle Calchaquí, se destaca el trabajo de Tarragó y De Lorenzi (1976), quienes describen cabezales líticos también hallados en contextos de superficie (ver Agnolin y Carbonelli 2017).

De todas formas, si bien los hallazgos en estratigrafía en sectores por debajo de los 3000 msnm aún esperan por ser descubiertos, esto no ha impedido la obtención de cronología absoluta. En este sentido, nuevas técnicas como aquella denominada varnish microlamination han sido aplicadas con buenos resultados, indicando cronologías correspondientes al Holoceno temprano y medio para el caso de artefactos de contextos de superficie (ver Somonte y Baied 2013, Carbonelli 2014). Siguiendo esta intención de documentar la presencia de ocupaciones humanas anteriores a los 3000 AP en sectores altitudinales por debajo de la puna, en este trabajo presentamos el análisis de un total de 28 artefactos "Saladillo", provenientes del Departamento de Cachi, valle Calchaquí, Provincia de Salta. Los mismos fueron registrados en archivo por parte de Pío Pablo Díaz y colaboradores, con fecha de 1986, y alojados entre las colecciones del Museo Arqueológico de Cachi, el cual lleva su nombre. En abril de 2016 surgió la posibilidad de revisar dichas colecciones, realizando un análisis que en parte se presenta en este trabajo.

Los artefactos Saladillo son motivo de comunicaciones científicas desde largo tiempo atrás, remontándose a los inicios del siglo XX, y tratándose en su mayoría de conjuntos recuperados en la puna (Boman [1908] 1991, Menghin 1954). Los mismos se caracterizan por presentar una morfología tendiente a lanceolada -dados sus bordes convexos que convergen desde la porción central hacia los extremos-, módulos laminares y empleo de la tecnología de hojas, entre otros rasgos (Restifo y Patané Aráoz 2017). Su presencia está bien datada en contextos de la puna de Jujuy y Salta, con fechas en torno al lapso de ca. 55004000 AP (Fernández 1983, Fernández Distel 2007, Muscio 2011, López y Restifo 2017, Restifo y Patané Aráoz 2017). Dichas fechas ubican a estos artefactos en el segmento de finales del Holoceno medio. Dado el lapso temporal acotado -considerando la escala temporal amplia- que reflejan los fechados de los artefactos "Saladillo", los mismos poseen un potencial relevante para aproximar cronología de modo relativo en conjuntos de artefactos arqueológicos provenientes de contextos no estratificados (López y Restifo 2017).

Entonces, el objetivo central de este trabajo es discutir la posibilidad de presencia de ocupaciones humanas anteriores a los $3000 \mathrm{AP}$ en el valle Calchaquí de la Provincia de Salta, sobre la base del estudio de la variabilidad del conjunto de artefactos Saladillo recuperado en el departamento de Cachi. Se destaca que se trata de uno de los pocos conjuntos de esta clase de artefacto detectados por fuera de la puna, lo que representa una oportunidad relevante para discutir la posibilidad de ocupaciones humanas correspondientes al segmento temporal del Holoceno medio en sectores de menor altitud.

En vistas del objetivo planteado se presenta un análisis de atributos cuantitativos y cualitativos de los artefactos en cuestión, orientado a la identificación de tendencias centrales y de dispersión. A su vez, se plantea una discusión retomando resultados de trabajos anteriores en base a conjuntos de la puna, adoptando una perspectiva comparativa, y apuntando a discutir posibles causas de la distribución de dicha clase artefactual en diferentes localidades del NOA.

\section{Cronología, generalidades y particularidades de los artefactos "Saladillo".}

En su cuadro cronológico del NOA Antonio Serrano propuso una fase "Saladillense", ubicada hacia los ca. $4000 \mathrm{AP}$, y visible tanto en puna como en el valle Calchaquí (Serrano 1967: 3). Si bien en este trabajo no se sigue una perspectiva de definición de fases, sí resulta relevante la cronología pensada por el autor, la cual mantiene coherencia con los fechados absolutos disponibles.

La cronología de los artefactos Saladillo se puede establecer a partir de la consideración de los siete fechados absolutos obtenidos hasta el momento, en sitios de la puna de Jujuy y de la puna de Salta. En la puna de Jujuy se dispone de dos fechados en dos sitios diferentes. Uno de ellos proviene del sitio Río Grande, el cual dio por resultado $5520 \pm 270 \mathrm{AP}$ (Fernández 1983: 80). El sitio restante es aquel denominado El Pasaje, cuya fecha es de 3995 土 75 AP (Fernández Distel 2007: 154).

En la puna de Salta, por su parte, se obtuvieron cinco fechados absolutos, provenientes de dos sitios. En primer lugar se destaca el sitio Alero Cuevas, cuyos fechados son $4210 \pm 70$ AP y $5206 \pm 68$ AP (López y Restifo 2017: 50). Se destaca que en este sitio, aparte del hallazgo de más de una decena de artefactos Saladillo, también se recuperó, asociado a los mismos, un núcleo con extracciones laminares así como desechos de talla relacionados con la talla laminar. Esto contribuyó a la comprensión de la variante tecnológica implementada para la manufactura de los artefactos en cuestión (López y Restifo 2012, Restifo 2015). A su vez, otros tres fechados fueron obtenidos a partir de los trabajos en el sitio Ramadas Perfil Norte. Los mismos dieron por resultado $5210 \pm 40 \mathrm{AP} ; 5273 \pm 71 \mathrm{AP}$ y $5333 \pm 40$ AP (Muscio 2011: 175). Entonces, a partir de las siete fechas absolutas presentadas, puede establecerse un rango cronológico entre los ca. 5500-4000 AP para los 
artefactos Saladillo, quedando ubicados en el segmento de finales del Holoceno medio.

Asimismo, considerando sus rasgos característicos, destacamos las tendencias centrales observadas. Las mismas constituyen una síntesis de los análisis presentados en trabajos anteriores, en los que se consideraron conjuntos de los sitios mencionados de la Puna de Salta, así como de conjuntos de superficie del sector salteño de Salinas Grandes (Patané Aráoz 2013, Restifo 2015, Restifo y Patané Aráoz 2017). Las tendencias centrales pueden resumirse del siguiente modo:

-Bajo grado de variación métrica en dimensiones de longitud, ancho y espesor (coeficientes de variación siempre por debajo de 0,25).

-Módulos laminares.

-Baja diversidad de rocas (por lo general cuarcitas y andesitas de orden local, sumadas a otras clases de roca que aparecen en muy bajas frecuencias).

-Forma base de hojas o lascas laminares.

-Mayor concentración de retoque en cara dorsal. Cuando el mismo se observa en cara ventral se relaciona con secuencias de lascado sobre el extremo proximal, que reducen el espesor del bulbo.

-Sección longitudinal planoconvexa.

-Retoque marginal

-Presencia de filos largos y puntas herramienta.

Desde luego, estas tendencias representan una abstracción realizada a nivel del conjunto, y de ningún modo debe considerarse que los especímenes de los artefactos Saladillo son idénticos entre sí. En los conjuntos se observan variaciones, como es de esperar (e.g. uso de lascas angulares como formas base, presencia de filos naturales, retoque extendido en cara dorsal, entre otras). Sin embargo, la presentación a partir de tendencias centrales favorece la organización de los datos y la comprensión de la variabilidad de las piezas que se tratan.

\section{Acerca del área de estudio y la muestra analizada}

Dentro del sistema de valles y quebradas que componen el denominado valle Calchaquí, el cual se extiende unos 400 km² abarcando sectores de las provincias de Salta, Catamarca y Tucumán, el sector Norte se caracteriza por la presencia de altitudes en el fondo de valle que oscilan entre los 3500 y 1680 msnm. Asimismo, destacan picos nevados que superan los 6000 msnm, como el nevado de San Martín, uno de los más elevados de la región. El régimen de precipitaciones es principalmente estival, oscilando entre los $100 \mathrm{~mm}$ y $200 \mathrm{~mm}$ anuales (Valencia y Lago 1970). Los valles y quebradas que integran el sistema pueden considerarse como pasos naturales que conectan el valle Calchaquí con pisos altitudinales de mayor elevación como la puna, y particularmente a los valles del sector salteño con la puna de la misma provincia (Williams y Villegas 2017). Entre dichos pasos puede mencionarse el propio valle del río Calchaquí, cuyas nacientes se localizan en las elevaciones del nevado de Acay (5716 m), puna de Salta (Valencia y Lago 1970).

Asimismo, dentro del sector norte del valle Calchaquí, el conjunto que motiva este trabajo se compone de 28 ejemplares de artefactos Saladillo. La mayor parte del conjunto fue recuperada en las inmediaciones de

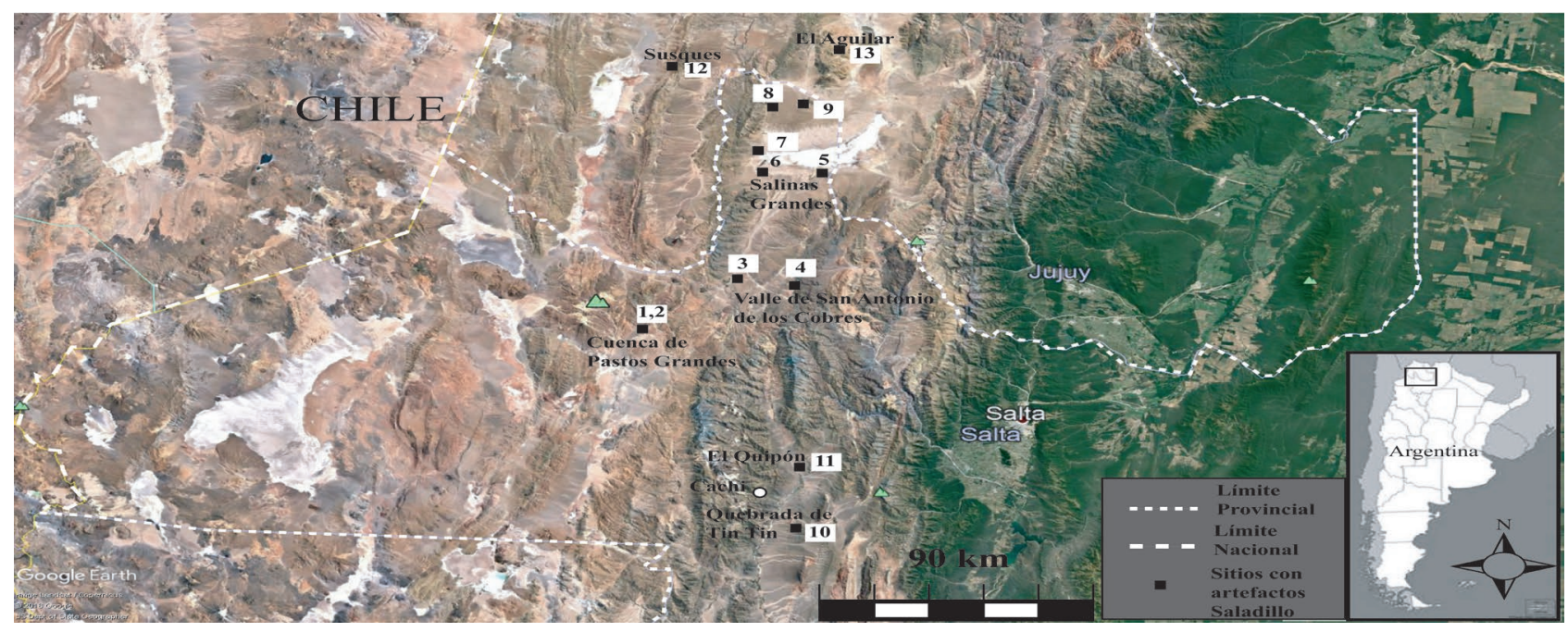

Figura 1. Ubicación del área de recolección del conjunto analizado y contexto regional incluyendo sitios con hallazgos de artefactos Saladillo de Salta y Jujuy. Referencias: 1-Alero Cuevas; 2- La Hoyada; 3- Ramadas Perfil Norte; 4- Sector oriental del valle de San Antonio de los Cobres; 5- Cangrejillos; 6- Niño Muerto; 7- Cobres; 8- Rangel; 9- Cerro Negro; 10- Quebrada de Tin Tin; 11- SSalCac 7; 12- Distribuciones de superficie en Susques; 13- Distribuciones de superficie en El Aguilar.

Figure 1. Analized assemblage collection area and regional context, higlighting sites with Saladillo artifacts founded in Salta and Jujuy. References: 1-Alero Cuevas; 2- La Hoyada; 3-Ramadas Perfil Norte; 4- Eastern side of San Antonio de los Cobres valley; 5- Cangrejillos; 6- Niño Muerto; 7- Cobres; 8-Rangel; 9-Cerro Negro; 10- Quebrada de Tin Tin; 11-SSalCac 7; 12-Surface scatters in Susques; 13- Surface scatters in El Aguilar. 
la quebrada de Tin Tin ( $n=24)$ (Figura 1), según consta en los ordenados y completos registros de Pío Pablo Díaz, disponibles en el Museo Arqueológico de Cachi, y con fecha de registro del 10 de octubre de 1986. Dicha quebrada se ubica en las cercanías de la localidad de Escalchi, a una altura promedio de 2500 msnm y a unos cinco kilómetros de la localidad de Cachi, dentro del departamento homónimo. El propio investigador, Pío Pablo Díaz, consignó en sus registros, y en relación a los hallazgos, la cronología "precerámica" entre otras. El componente restante de la muestra $(n=4)$, fue recuperado en la localidad El Quipón, también por Pío Pablo Díaz, y a unos $10 \mathrm{~km}$ al Norte de la quebrada de Tin Tin, en el sitio denominado SSalCac 7, según la nomenclatura propia del Museo Arqueológico de Cachi.

\section{Metodología}

Se realizaron diferentes mediciones sobre cada artefacto lítico del conjunto, las que apuntaron a su descripción morfológica general. De este modo, se identificaron tendencias centrales y de dispersión a nivel de conjunto, las que contribuyeron a la comprensión de la variabilidad. Los atributos considerados, tomados de la propuesta de clasificación de uso recurrente en Argentina (Aschero 1975, 1983), así como de otras propuestas (Cardillo 2002; López 2008), fueron las siguientes: 1) longitud, ancho y espesor, medidas en milímetros con calibre; 2) clase de roca; 3) forma base; 4) sección longitudinal; 5) situación de los lascados sobre las caras; 6) extensión de los lascados sobre las caras; 7) cantidad de filos y/o puntas por artefacto. A su vez, y especialmente vinculadas al análisis de filos, se seleccionaron las restantes variables: 8) extensión relativa sobre el borde; y 9) presencia de filos naturales con rastros complementarios. Asimismo, se presentó la clasificación según grupos tipológicos (Aschero 1975, 1983).

Para el caso de longitud, ancho y espesor se realizó su presentación mediante estadística descriptiva, considerando la media, desvío estándar y valores mínimo y máximo. Se utilizó el software PAST 2.4 (Hammer et al. 2001), de uso gratuito. A su vez, se informó el coeficiente de variación (CV), el cual representa una medida de la heterogeneidad de los valores de un conjunto de casos para una variable determinada. Su resultado puede variar de cero a uno. Cuanto más cerca de uno la heterogeneidad es cada vez mayor. Entonces, valores bajos de CV expresan baja variación (Cardillo 2002; López 2008). El coeficiente se calcula dividiendo el desvío estándar por la media, es decir:

$\mathrm{CV}=\mathrm{s} / \mathrm{m}$

En relación con la situación de los lascados sobre las caras, empleamos las categorías "unifacial" y "bifacial". Aclaramos que en la perspectiva de análisis seguida, el uso de ambos términos es independiente de la extensión que puedan tener los lascados sobre cada una de las caras y de las clases técnicas que involucren (ver Hocsman 2006), aludiendo únicamente al hecho de que un artefacto puede presentar talla en una de sus caras o en ambas. La descripción del trabajo realizado en cada cara la consideramos como un paso posterior a la distinción inicial entre unifacial y bifacial.

Para el caso de la variable "cantidad de filos y/o puntas por artefacto", la cuantificación se basó en su conteo por cada uno de los artefactos. Dado que varios presentan más de un filo y/o punta, el número total superó al de los propios artefactos que componen cada conjunto, lo que se verá reflejado en la tabla correspondiente. Particularmente, se destaca que con el término filo se alude a secuencias de lascados continuos mayormente concentradas en el borde de una pieza, definiendo una arista. Asimismo, el término punta se refiere a las denominadas "puntas herramientas", aquellas formatizadas en un sector del artefacto, definidas por la convergencia de dos aristas que dan lugar a un ápice activo y que, en términos hipotéticos, se vincularían a funciones diferentes de las extractivas (caso de las puntas de proyectil) (Aschero 1975:19).

Por último, siguiendo a diferentes autores, cuando se habla de hojas líticas hacemos referencia a artefactos que presentan un patrón de atributos específicos distintivos, tanto por sus dimensiones de longitud y ancho como por los atributos de las caras (Aschero 1975; Bar-Yosef y Kuhn 1999). Así, se destaca la morfología alargada, caracterizada por una relación en la que la longitud corresponde al doble de la medida del ancho o más, entendida como módulo laminar (Aschero 1975). Se destaca también la presencia de bordes rectos paralelos o subparalelos, y de aristas rectas paralelas o subparalelas en cara dorsal, lo que indica extracciones anteriores. En general, el eje técnico, entendido como aquel que divide en dos partes iguales al talón, se presenta de manera paralela a los negativos de extracción y aristas. A su vez, se diferencia a las hojas de las lascas laminares. Estas últimas tienen la particularidad de no presentar en su cara dorsal un patrón de aristas paralelas o subparalelas, sino que pueden contemplar aristas en patrones oblicuos o angulares.

\section{Resultados}

El conjunto total analizado se compone de 28 artefactos, de los cuales 14 (50\%) se encuentran en estado entero. Una muestra de los artefactos analizados se presenta en la figura 2. Tal como se indicó en el apartado de metodología, el análisis contempló atributos cuantitativos y cualitativos. El análisis cuantitativo incluyó los atributos de longitud, ancho y espesor (Tabla 1), tomando en cuenta los artefactos enteros. Se observó un bajo grado de variación métrica, tomando en cuenta especialmente el coeficiente de variación (CV). El mismo alcanza un valor máximo de 0,17 según se observa para los casos de ancho y espesor. Por su parte el atributo longitud presenta un valor levemente menor, de 0,15. Estos resultados de 
coeficientes indican una baja heterogeneidad de valores en las medidas de los artefactos. A su vez, si bien los valores mínimo y máximo de longitud, ancho y espesor dan cuenta de rangos de variación considerable, son destacables los bajos valores de desvío estándar, que indicarían distribuciones concentradas en torno a la media.

Por su parte, en relación con los atributos cualitativos, en la tabla 2 se presentan las tendencias para cada atributo. Entre las clases de rocas se destaca el predominio claro de la cuarcita, seguida luego por la andesita. A su vez, bajo el rótulo "otras" se incluyen algunos ejemplares cuya materia prima aún espera por ser determinada con exactitud, y que engloba rocas de grano fino en tonalidades gris, marrón y verde. De todas formas, se destaca que sumando las frecuencias relativas de andesita y cuarcita, las mismas representan más del $70 \%$ del conjunto. Esto podría reflejar una tendencia hacia una baja diversidad de rocas.

Respecto de la forma base, se decidió incluir a hojas y lascas laminares en una misma categoría. La razón de esta inclusión se debió a la dificultad para su distinción, a causa de fracturas en las piezas, o bien secuencias de retoque invasivas sobre la cara dorsal, lo que dificultó la observación de atributos diagnósticos. La tendencia identificada indica el claro predomino de hojas o lascas laminares como forma base (43\%). Es interesante, a su vez, la observación de un caso de empleo de lasca, dado un notorio desfasaje entre eje técnico y morfológico, y presencia de un patrón de aristas en disposición angular. Esto refleja variabilidad en el empleo de formas base para manufacturar artefactos Saladillo, aparte de la tecnología laminar. En relación a la sección longitudinal se observó la presencia absoluta de la variante planoconvexa, destacándose caras ventrales planas y caras dorsales con cierta curvatura, a causa del trabajo de retoque, siempre que se observen las piezas en norma longitudinal.

Por su parte, considerando la situación de los lascados sobre las caras, se observó el predominio de la talla bifacial (54\%), por encima de la unifacial (14\%), mientras que en los restantes casos la presencia de fracturas impidió la apreciación. Se destaca que el trabajo sobre cara dorsal incluye la formatización de filos en mayor medida. En la cara ventral se registraron 14 casos (50 \%) de presencia de retoque concentrado en el extremo proximal, generando la reducción del espesor del bulbo, tal como fuera observado en muestras de la puna (ver detalle de piezas 1 y 6 en figura 2). En seis casos (21\%) el bulbo no presentó alteraciones, y en los ocho casos restantes (29\%) no pudo apreciarse a causa del estado de fragmentación. A su vez, respecto de la extensión de los lascados sobre las caras, la talla marginal, ya sea como única modalidad de talla o bien en combinación con la talla parcialmente extendida, resultó ser la de mayor representación. Es decir, puede plantearse la presencia de artefactos con tendencia al retoque bifacial y marginal en mayor medida. Asimismo, debe destacarse la representación de la talla parcialmente extendida, lo que indica mayor variabilidad en el trabajo sobre las caras de las piezas, expresándose, si bien en casos aislados, en ambas caras de un artefacto.

Tomando en cuenta lo relativo a filos, se observó el predominio notorio de los artefactos de dos y tres filos, los cuales presentan frecuencias similares, y que en conjunto alcanzan el $65 \%$ de la muestra de artefactos analizada. Los artefactos de uno y cuatro filos están representados, aunque en baja frecuencia, tratándose de un ejemplar en cada caso. Considerando la extensión relativa de

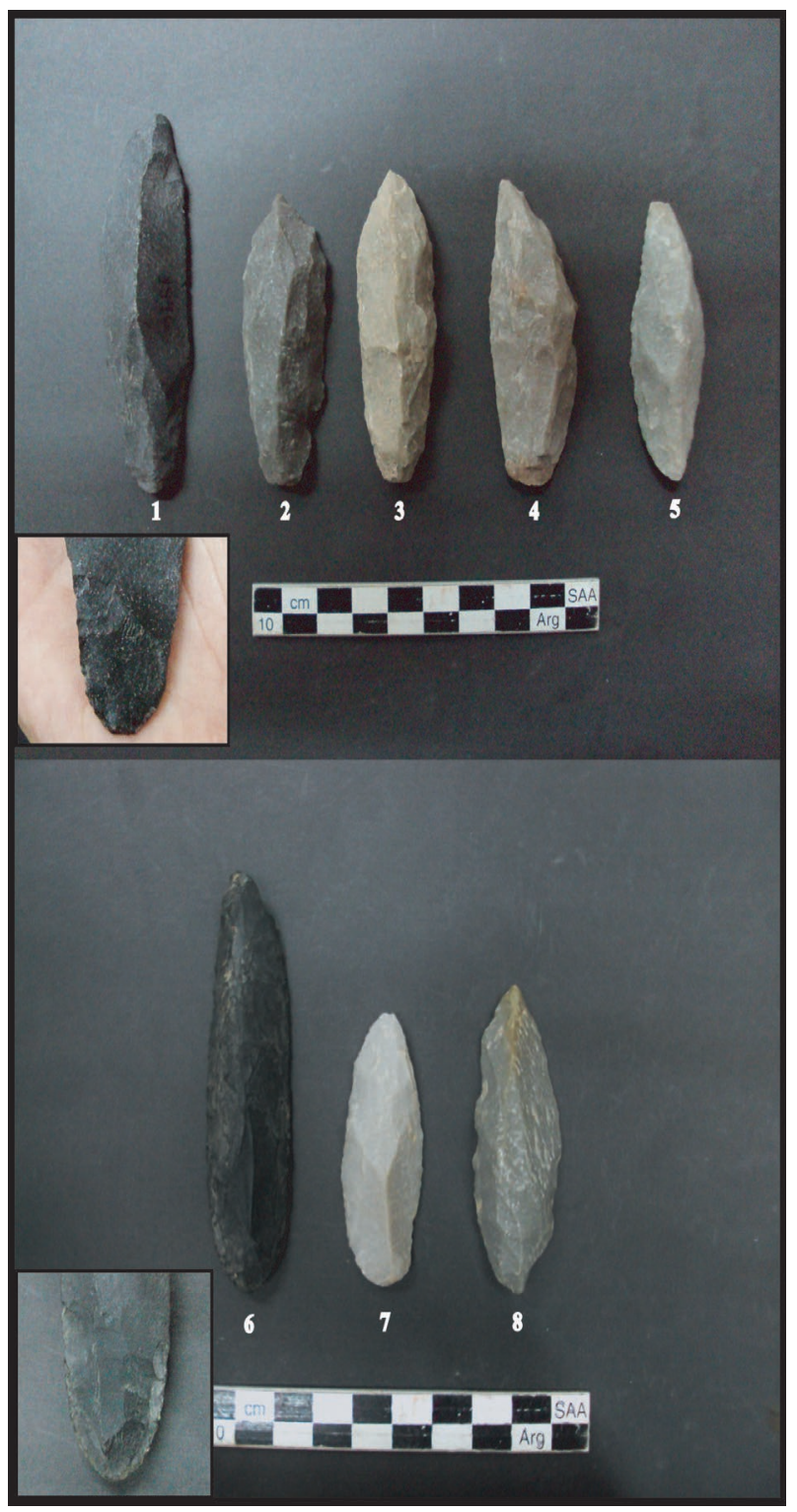

Figura 2. Artefactos Saladillo recuperados en el sector Norte del valle Calchaquí. Detalle del las piezas 1 y 6 , vistas en cara ventral y destacándose el retoque que reduce el espesor del bulbo.

Figure 2. Saladillo artifacts recovered at the Northern Calchaqui Valley. Zoom of pieces 1 and 6 from its ventral face, and highlighting the retouch that reduce the bulb's thickness. 
los filos sobre el borde de las piezas, se observó una marcada tendencia hacia artefactos con presencia de filos largos. En este sentido, de los 44 filos indentificados, 29 corresponden a la categoría largo (66\%), mientras que apenas cuatro se clasificaron como cortos. A su vez, se destaca la presencia de siete casos de filo natural con rastros complementarios (FNRC).

Como última instancia de esta sección de resultados, se presenta lo relativo a grupos tipológicos (Tabla 3) combinados con la presencia de FNRC. Se destaca un patrón de predominio de raederas, ya sea en artefactos de dos, tres o cuatro filos y/o puntas o bien en combinación con los FNRC. Por su parte, las puntas herramienta son las que siguen en orden de frecuencia. Se destaca también la ausencia del grupo tipológico cuchillo, rasgo que había sido identificado en los conjuntos de la puna.

En resumen, las tendencias centrales destacadas a partir de los resultados expuestos indican la presencia, a nivel del conjunto, de: 1) artefactos de baja variación en sus medidas de longitud, ancho y espesor (valores de CV menores a 0,17$)$; 2) manufacturados principalmente en cuarcita y andesita; 3) empleando hojas o lascas laminares

\begin{tabular}{lccccc}
\hline & Media & D. Estándar & Valor min. & Valor max. & CV \\
\hline Longitud & 64,5 & 9,88 & 46,6 & 84,3 & 0,15 \\
Ancho & 24,19 & 4,13 & 35 & 19,5 & 0,17 \\
Espesor & 12,08 & 2,09 & 8,2 & 16,7 & 0,17 \\
\hline
\end{tabular}

Tabla 1. Estadística descriptiva para las dimensiones de longitud, ancho y espesor. Valores en milímetros.

Table 1. Length, width and thickness descriptive stats. Values in milimeters.

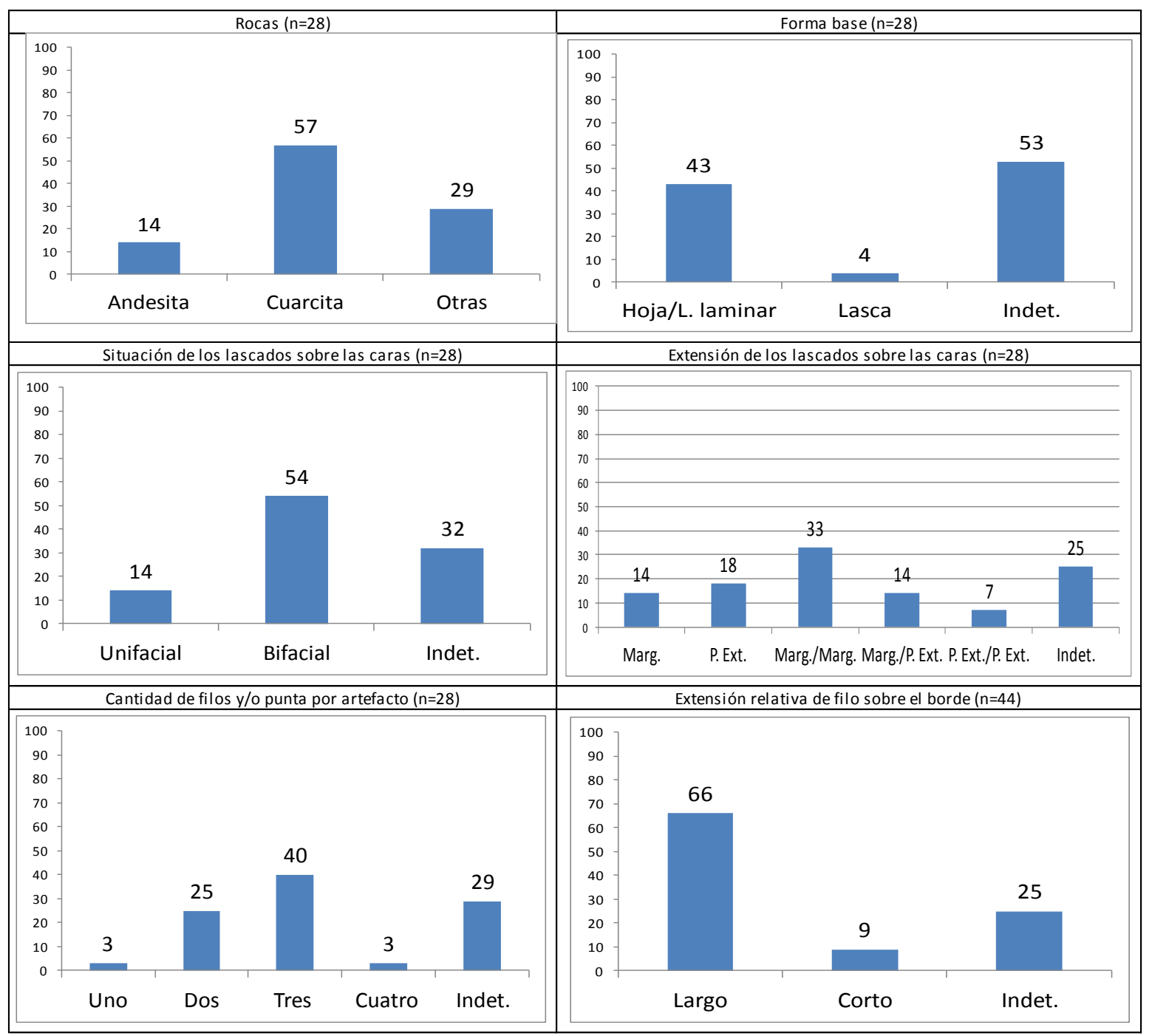

Tabla 2. Tendencias de los atributos cualitativos (frecuencias relativas).

Table 2. Tendencies of cualitative attributes (relative frequencies). 


\begin{tabular}{lcc}
\hline \multicolumn{1}{c}{ Grupo tipológico / FNRC } & $\mathrm{n}$ & $\%$ \\
\hline Punta herramienta + FNRC + FNRC & 1 & 3,5 \\
Raedera + FNRC & 1 & 3,5 \\
Raedera + Raedera + FNRC & 1 & 3,5 \\
Raedera + Raedera & 4 & 14,5 \\
Raedera + Raedera + cortante & 1 & 3,5 \\
Raedera + Raedera + Punta herramienta & 8 & 29 \\
Raedera + Raedera + P. herram + FNRC & 1 & 3,5 \\
Raedera + Raedera + P. herram. + P. herram. & 1 & 3,5 \\
FNRC + FNRC & 1 & 3,5 \\
FNDAF & 9 & 32 \\
\hline Total & 28 & 100 \\
\hline
\end{tabular}

Tabla 3. Grupos tipológicos y filos naturales con rastros complementarios (FNRC).

Table 3. Typological groups and edges with scars (FNRC).

la manufactura de esta clase de artefacto, lo que ha sido observado hasta ahora en todos los conjuntos del NOA. De todas formas, la obsidiana es una roca que aparece acompañando los conjuntos de artefactos Saladillo de la puna, estando representada en otros artefactos de filo retocado (sobre lascas),

como forma base; 4) sección longitudinal planoconvexa en todos los casos; 5) patrón de talla bifacial y 6) marginal; 7) presencia de dos o tres filos; 8) largos; 9) correspondientes al grupo tipológico raedera. Como tendencias de dispersión respecto del patrón señalado se destaca el uso de lasca como forma base en un caso; artefactos con retoque parcialmente extendido; artefactos con un único filo largo o punta herramienta, o bien, que combinan filo largo con punta herramienta o FNRC.

\section{Discusión y conclusiones}

Siguiendo el objetivo central de este trabajo, dirigido a discutir la posibilidad de presencia de ocupaciones humanas anteriores a los 3000 AP en el sector norte del valle Calchaquí, se consideran los resultados de análisis obtenidos tomando los antecedentes mencionados de estudios de conjuntos de artefactos Saladillo provenientes de la puna de Jujuy y Salta.

Retomando los rasgos predominantes en los conjuntos de artefactos Saladillo, presentados en la sección dedicada a la "cronología, generalidades y particularidades", puede realizarse una comparación entre los conjuntos recuperados en la puna, y el conjunto proveniente del sector Norte del valle Calchaquí, motivo principal de este trabajo. En este sentido, se destaca el bajo grado de variación métrica observado en el conjunto proveniente del departamento de Cachi, el cual se encuentra por debajo del valor de CV de 0,25 observado en conjuntos de la puna. Asimismo, el predominio de hojas o lascas laminares como formas base es coherente con lo observado en la puna. Otra similitud a destacar es la baja diversidad de rocas empeladas para su manufactura. Debe remarcarse que en el caso de la puna siempre se trata de materias primas locales. Si bien aún no se determinó con exactitud la procedencia de las rocas del conjunto analizado, es muy probable que sean de orden local también, dada la geología regional. En este sentido se destaca la presencia de la formación Pirgua en la región. La misma constituye un sub-grupo de la formación Salta y se expresa a modo de areniscas y conglomerados, incluyendo clastos de cuarcita, entre otros (Hongn s/f).

A su vez, es notable la ausencia de obsidiana para puntas de proyectil, así como en desechos de talla (Restifo 2015). Una posible explicación para la exclusión de dicha roca en la manufactura de artefactos Saladillo podría ser el hecho de una selección hacia rocas con una menor propensión al quiebre, lo que ha sido interpretado en el marco de una estrategia tecnológica con tendencia a diseños confiables, apuntando a artefactos eficientes en su función (ver Restifo y Patané Aráoz 2017).

La mayor concentración del retoque en cara dorsal, así como la presencia de retoque en cara ventral, orientado a la reducción del espesor del bulbo, es otro rasgo similar entre los conjuntos de la puna, y el conjunto del sector Norte del Valle Calchaquí. Dicha tarea de reducción del espesor del bulbo estaría orientada a generar caras ventrales totalmente planas, lo que probablemente pudo haber sido favorable para su enmangue (López y Restifo 2012). En este sentido, es relevante destacar la presencia absoluta de secciones longitudinales planoconvexas en todos los conjuntos. A su vez, otro rasgo en común es el predominio de retoque marginal. El mismo se habría orientado a la formatización de filos largos asignables al grupo tipológico raedera, atributo también predominante tanto en los conjuntos de la puna como en el conjunto del sector Norte del valle Calchaquí.

Ahora bien, ¿qué implicancias tienen estas similitudes entre conjuntos de artefactos Saladillo recuperados en la puna y más allá, en el sector Norte del valle Calchaquí? En principio, podemos sostener que la recurrencia de atributos observados en todos los conjuntos permite clasificarlos en una misma categoría, o bien clase (O'Brien y Lyman 2002), que en este caso es la de Artefactos Saladillo. Clase, en este sentido, implica el hecho de especificar un conjunto de atributos que definen la inclusión de un especímen bajo su rótulo (Dunnell 1977), definida la clase Saladillo por los atributos listados al inicio del trabajo (sección en torno a generalidades y particularidades). Considerando esta clasificación bajo una misma categoría como punto de partida, y retomando la cronología absoluta que en la puna ha sido asignada a los artefactos que integran la clase en cuestión, es lógico pensar una cronología similar de los artefactos provenientes del sector Norte del valle Calchaquí, en relación con aquella establecida para la puna. En consecuencia, los mismos pueden 
ser indicadores del segmento temporal de finales del Holoceno medio. Por lo tanto, la presencia de artefactos Saladillo sería un reflejo parcial de ocupaciones humanas de dicha cronología en el sector Norte del valle Calchaquí. En ausencia total de fechados absolutos para dicho sector, los artefactos Saladillo se convierten en un primer indicador cronológico -si bien relativo- para la región.

Debe destacarse que el empleo de artefactos líticos para establecer cronologías relativas ha resultado una opción confiable, especialmente para pensar la dimensión temporal de palimpsestos de superficie (Jones y Beck 1992). Para el caso de Argentina, vale como ejemplo el caso de las puntas denominadas "Bird IV", asignadas al bloque temporal del Holoceno tardío, en el marco de la arqueología patagónica (Franco et al. 2005). A su vez, considerando una escala espacial de mayor amplitud, es conocido el caso de las puntas de proyectil "cola de pescado", asociadas a fechados absolutos en diferentes sitios del continente americano (Miotti y Terranova 2015), y consideradas un indicador relevante representativo de cronologías de la transición Pleistoceno-Holoceno y Holoceno temprano.

Entonces, sobre la base del marco cronológico propuesto, considerando a los artefactos Saladillo del sector Norte del valle Calchaquí como indicador de ocupaciones humanas de finales del Holoceno medio, se plantea otro interrogante: ¿Qué procesos de orden cultural habrían posibilitado la distribución de esta clase de artefacto tanto en el territorio puneño como más allá del mismo, en el sector de valles? En principio, puede pensarse que esta distribución de los artefactos Saladillo sería la señal arqueológica (o bien una de ellas) de desplazamientos de grupos humanos entre diferentes sectores altitudinales del NOA. Suponiendo que las rocas de los artefactos de la puna son exclusivas de dicho sector, y lo mismo para el caso del sector de valles, no se invalida la proposición. Esto, debido a que un grupo puede desplazarse con artefactos así como con ideas, o bien saberes (Franco et al. 2010), que luego guían la replicación de variantes tecnológicas sobre la base de las materias primas de otros espacios ocupados.

Otra posible explicación para la distribución de los artefactos Saladillo más allá de la puna, es algún grado de conexión entre poblaciones de dichos sectores, implicando contactos entre grupos (Restifo y Patané Aráoz 2017). Esto habría posibilitado la transmisión de información, lo que pudo haber ocurrido a la par de la circulación de otros elementos como materias primas vegetales, de las que se conoce registro (Rodríguez 2004). De todas formas, también habría implicado desplazamientos. La transmisión de información entre grupos humanos ha sido propuesta como un rasgo relevante para la adaptación humana, permitiendo la incorporación de variantes tecnológicas mediante el aprendizaje, ya sea por imitación o bien ensayo y error
(Boyd y Richerson 1985, Bettinger y Eerkens 1999, Fitzhugh 2001, Franco et al. 2005), e incluyendo la observación de los resultados del uso de la variante tecnológica adoptada. La posibilidad de existencia de fuertes sesgos -o baja tolerancia a la variación- (Boyd y Richerson 1985, Cardillo 2002) en la replicación de esta clase artefactual, implicando instrucciones o procedimientos precisos, puede evidenciarse en las regularidades de los atributos de los artefactos Saladillo, tales como su baja variación métrica, el absoluto predominio de secciones planoconvexas, la reducción del espesor del bulbo, entre otros rasgos observados en los conjuntos de puna y ahora también de valles.

La propia topografía del NOA habría favorecido la posibilidad de circulación entre valles y puna debido a la presencia de "pasos naturales" que conectan pisos altitudinales (Williams y Villegas 2017). En este sentido, se destaca el propio valle del río Calchaquí, cuyas nacientes se ubican en las alturas del nevado de Acay, en plena puna. Como trabajo a futuro será relevante relevar el registro arqueológico de dichos pasos atendiendo a la presencia de artefactos diagnósticos de cronologías más tempranas que los 3000 AP. En este sentido, tanto los artefactos Saladillo como diferentes clases de puntas de proyectil pueden resultar valiosos.

Asimismo, se aclara que cuando se considera la posibilidad de desplazamiento de grupos entre pisos altitudinales diferentes, puede considerarse la movilidad de tipo estacional, como ha sido planteado (Yacobaccio 1991), aunque también puede considerarse una frecuencia de movimientos -o bien contactos entre poblaciones distantes- menor que la anual, pero suficiente para dejar una impronta en el registro arqueológico, es decir en los palimpsestos formados en el largo plazo. Esto se plantea sobre la base de un concepto de registro arqueológico "promediado", lo que significa que aquellos eventos de mayor recurrencia en el tiempo (en escalas de diversa amplitud) son los que dejan una huella material visible a modo de agregado (Borrero 1993).

Si bien los interrogantes planteados pueden ser respondidos de manera tentativa por el momento, no debe soslayarse su relevancia en tanto implican un inicio de integración de resultados obtenidos en los diferentes sectores altitudinales del NOA, que en lo relativo a cronologías anteriores a los $3000 \mathrm{AP}$ no ha sido recurrente. A su vez, dentro de estos interrogantes, debe considerarse que la cronología relativa propuesta posee cierta robustez, dados los fechados de los artefactos Saladillo de la puna y las recurrencias de atributos entre conjuntos de tal sector y de valles, tal como se presentó aquí. Este criterio de similitud para establecer cronologías, es aceptable en ausencia de otras posibilidades. A su vez, los Artefactos Saladillo no son la única clase con potencial "tiempo sensitivo". Al menos en la puna argentina, y con rangos temporales un poco más amplios, otras clases de 
artefactos poseen dicho potencial, tales como las puntas de proyectil triangulares apedunculadas, extendidas en el rango de ca. 10000-8000 AP, entre otras (Hoguin 2014, López y Restifo 2017), o bien puntas de proyectil lanceoladas, correspondientes al bloque del Holoceno medio mayormente, e inicios del Holoceno tardío. Estas últimas también empleadas para aproximar cronologías en el sector de valles (Agnolin y Carbonelli 2017).

Por último, se destacan algunas particularidades de los artefactos Saladillo del sector Norte del valle Calchaquí, y en relación a lo observado en los conjuntos de la puna. En este sentido se observó la presencia de lasca como forma base, y la ausencia del grupo tipológico cuchillo. Estos rasgos constituyen particularidades, por el momento, de los artefactos del sector de valles. De todas formas, dado lo incipiente de la problemática planteada, las futuras investigaciones y aumento de las muestras permitirán confirmar esta observación constituyendo esto un patrón, o bien descartarla. Asimismo, es relevante considerar que esta clase artefactual no ha sido reportada para el caso de la puna de Catamarca, ni para el sector de valles de dicha provincia (Aschero y Hocsman 2011, Agnolin y Carbonelli 2017). Esto puede indicar orientaciones diferentes en la toma de decisión tecnológica generando un patrón de divergencia cultural visible en escala espacial amplia.

Tal como se planteó al principio de este trabajo, el objetivo central apuntó a discutir la presencia de ocupaciones humanas anteriores a los ca. 3000 AP en el sector norte del valle Calchaquí. Sobre una base de cronología relativa brindada por la presencia de artefactos Saladillo, se sostiene que en dicho sector existe evidencia de ocupaciones humanas correspondientes a finales del Holoceno medio. El planteo de interrogantes que expliquen la presencia de esta clase de artefactos tanto en puna como en valles significa una guía sobre la cual continuar la investigación en torno a la temática de las poblaciones cronológicamente tempranas en el amplio espacio del Noroeste Argentino.

Salta, 7 de febrero de 2018

\section{Agradecimientos}

Este trabajo fue posible gracias al apoyo económico del CONICET (Consejo Nacional de Investigaciones Científicas y Técnicas, Argentina) para la realización del proyecto de carrera de investigador en el marco del cual se realizó este trabajo. Un agradecimiento a las autoridades del Museo Arqueológico de Cachi así como a todo su personal, por su total predisposición para recibir investigadores/ as y facilitarles el acceso a las colecciones de materiales arqueológicos y a bibliografía disponible en su valiosa biblioteca. Especialmente al Lic. Jorge Cabral por su gran ayuda en el laboratorio.

\section{Bibliografía}

Agnolin, A. y J.P. Carbonelli. (2017). Diseños de puntas de proyectil en el valle de Santa María (Catamarca, Argentina): Una aproximación a la ocupación cazadora - recolectora. Chungara, 49 (4), 511-527.

Aschero, C. (1975). Ensayo para una clasificación morfológica de artefactos líticos aplicada a estudios tipológicos comparativos. Informe presentado al Consejo Nacional de Investigaciones Científicas y Técnicas (CONICET). M.S.

Aschero, C. (1983). Ensayo para una clasificación morfológica de artefactos líticos. Apéndice A y B. Cátedra de Ergología y Tecnología. Facultad de Filosofía y Letras. Universidad de Buenos Aires. M.S.

Aschero, C. y S. Hocsman. (2011). Arqueología de las ocupaciones cazadoras-recolectoras de fines del Holoceno medio de Antofagasta de la Sierra (Puna Meridional Argentina). Chungara, 43 (volumen especial), 393-411.

Bar-Yosef, O. y Kuhn, S. (1999). The big deal about blades: Laminar technology and human evolution. American Anthropologist, 101, 322-328.

Bettinger, R. y Eerkens, J. (1999). Point typologies, cultural transmission, and the spread of bow and arrow technology in the prehistoric Great Basin. American Antiquity, 64 (2), 231-242.

Boman, E. ([1908] 1991). Antigüedades de la región andina de la República Argentina y el desierto de Atacama. Tomos I y II. Jujuy: Universidad Nacional de Jujuy.

Borrero, L. (1993). Artefactos y evolución. Palimpsesto, 3, 15-32.

Boyd, R. y P. Richerson. (1985). Culture and the evolutionary process. Chicago: University of Chicago Press.

Carbonelli, J.P. (2013). El sitio Ampajango, 50 años después. Arqueología, 59 (1), 41-64.

Carbonelli, J.P. (2014). Evidencias paleoambientales y de producción lítica en la cantera taller Ampajango, Valle de Yocavil (Catamarca, Argentina). La Zaranda de Ideas, 9, 11-26.

Cardillo, M. 2002. Transmisión cultural y persistencia diferencial de rasgos. Un modelo para el estudio de la variación morfológica de las puntas de proyectil lanceoladas de San Antonio de los Cobres, Provincia de Salta, Argentina. En G. Martínez y J.L. Lanata (Eds.), Perspectivas integradoras entre Arqueología y Evolución (pp. 97-119). Olavarría: INCUAPA, Universidad Nacional del Centro.

Cigliano E., Bereterbide, S., Carnevali, B., Lorandi, A. M., y Tarragó, M. (1962). "El Ampajanguense". Publicación del Instituto de Antropología, 5, 7-104.

Dunnell, R. (1977). Prehistoria moderna. Introducción sistemática al estudio de la arqueología prehistórica. Madrid: Ediciones Istmo.

Fernández, J. (1983). Río Grande. Exploración de un centro precerámico en las altas montañas de Jujuy, Argentina. Ampurias, 45/46, 54-83.

Fernández Distel, A. (2007). El yacimiento de Guayatayoc (Jujuy, Argentina): Sus materiales líticos y un fechado de radiocarbono 
inédito. Cuadernos de la Universidad Nacional de Jujuy, 32, 151-166.

Fitzhugh, B. (2001). Risk and invention in human technological evolution. Journal of Anthropological Archaeology, 20, 125167.

Franco, N., Cardillo, M. y Borrero, L.A. (2005). Una primera aproximación a la variabilidad presente en las puntas denominadas "Bird IV". Werken, 6, 1-15.

Franco, N., Gómez Otero, J., Guráieb, G., Goye, S., Cirigliano, N. y Banegas, A. (2010). Variaciones espaciales en diseños de puntas pedunculadas medianas en patagonia argentina: una nueva aproximación. En R. Bárcena y H. Chiavazza (Eds.), Arqueología argentina en el bicentenario de la revolución de Mayo (pp. 283-287). Mendoza: Universidad Nacional de Cuyo.

Hammer, Ø., Harper, D. y Ryan, P. (2001). PAST: Paleontological statistics. Recuperado de www.toyen.uio.no/ ohammer/past.

Hernández Llosas, I. (2001). Quebradas Altas de Humahuaca a través del tiempo: el caso Pintoscayoc. Estudios Sociales del NOA, 2, 167-224.

Hocsman, S. (2006). Tecnología lítica en la transición de cazadores recolectores a sociedades agropastoriles en la porción meridional de los Andes Centro Sur. Estudios Atacameños, 32, 59-73.

Hocsman, S., Somonte, S., Babot, M., Martel, A. y Toselli, A. (2003). Análisis de los materiales líticos de un sitio a cielo abierto del área valliserrana del NOA: Campo Blanco, Tucumán. Cuadernos de la Facultad de Humanidades y Ciencias Sociales, 20, 325-350.

Hoguin, R. (2014). Secuencia cronológica y tecnología lítica en la Puna Seca y Salada de los Andes Centro-Sur para el Holoceno temprano y medio a través del ejemplo de Susques. Relaciones de la Sociedad Argentina de Antropología, 39, 333-364.

Hongn, F. D. (s/f). Hoja geológica 2566-III Cachi. Provincias de Salta y Catamarca. República Argentina. Salta: SEGEMAR.

Jones, G. y Beck, CH. (1992). Chronological resolution in distributional archaeology. En J. Rossignol y L. Wandsnider (Eds.), Space, Time and Archaeological Landcapes (pp. 167- 192). Nueva York: Springer.

López, G. (2008). Arqueología de cazadores y pastores en tierras altas. Ocupaciones humanas a lo largo del Holoceno en Pastos Grandes, Puna de Salta, Argentina. Oxford: BAR international series.

López, G. y Restifo, F. (2012). The Middle Holocene domestication and intensification of camelids in north Argentina, tracked by zooarchaeology and lithics. Antiquity, 86, 1041-1054.

López, G. y Restifo, F. (2017). El sitio Alero Cuevas, Puna de Salta, Argentina: Secuencia de cambio en artefactos líticos y resolución cronológica macrorregional durante el Holoceno temprano y medio. Chungara, 49 (1), 49-63.

Martínez, J., Mauri, E., Mercuri, C., Caria, E. y Oliszewski, N. (2013). Mid-Holocene human occupation in Tucumán (Northwest Argentina). Quaternary International, 307, 86-95.
Miotti, L., y Terranova, E. (2015). A hill full of points in terra incognita from Patagonia: notes and reflections for discussing the way and tempo of initial peopling. PaleoAmerica, 1(2), 181-196.

Menghin, O. (1954). Culturas Precerámicas en Bolivia. Runa, $6,125-132$

Muscio, H. (2011). Ocupaciones humanas a cielo abierto de finales del Holoceno medio y comienzos del Holoceno tardío en el Valle de San Antonio de los Cobres, Puna de Salta. Comechingonia, 15, 71-90.

O'Brien, M. y Lyman, L. (2002). The epistemological nature of archaeological units. Anthropological Theory, 2 (1), 37-56.

Patané Aráoz, J. (2013). Prospecciones arqueológicas en Salinas Grandes (Departamento de La Poma, provincia de Salta) y reporte de una punta "cola de pescado". Relaciones de la Sociedad Argentina de Antropología, 38 (1), 247-255.

Restifo, F. (2015). Tecnología de hojas líticas en tierras altas andinas: Perspectivas desde la Puna de la Provincia de Salta (Argentina). Estudios Atacameños, 51, 33-51.

Restifo, F. y Patané Aráoz, J. (2017). Artefactos Saladillo de la puna de la provincia de Salta (Argentina): Interpretación del proceso de cambio tecnológico hacia fines del Holoceno medio a partir de su clasificación funcional macroscópica. Relaciones de la Sociedad Argentina de Antroplogía, 42 (1), 13-34.

Restifo, F., Carbonelli, J.P., y Agnolin, A. (2018). Puntas de proyectil de puna y valles mesotermales del Noroeste Argentino en perspectiva comparativa: Los casos de la cuenca de Pastos Grandes (departamento de los Andes, Salta) y el valle de Santa María (Catamarca) como aporte para la arqueología de cazadores recolectores. Revista Andes. En prensa

Rodríguez, M. (2004). Cambios en el uso de los recursos vegetales durante el Holoceno en la Puna Meridional argentina. Chungara, 36, 403-413.

Serrano, A. (1967). Historia Cultural del Tucumán Prehispánico. Ampurias, 29, 1-90.

Tarragó, M. y De Lorenzi, M. (1976). Arqueología de los valles Calchaquíes. Etnía, 1, 1-35

Somonte, C. y Baied, C. (2013). Edad mínima de exposición de superficies en canteras-taller: reflexiones en torno a las primeras dataciones mediante microlaminaciones del barniz de las rocas (VML) para el noroeste argentino. Chungara, 45 (3), 427-445.

Valencia, R. y Lago, A. (1970). Relación suelo - geomorfología en el valle Calchaquí, provincia de Salta, República Argentina. Revista de la Asociación Geológica Aergentina, 25 (1), 71-85.

Williams, V. y Villegas, P. (2017). Rutas y senderos prehispánicos como paisajes. Las quebradas altas del valle Calchaquí medio. Boletín del Museo Chileno de Arte Precolombino, 22 (1), 71-94.

Yacobaccio, H.D. (1991). Sistemas de asentamiento de los cazadores recolectores tempranos en los Andes Centro Sur. Tesis doctoral. Universidad de Buenos Aires, Buenos Aires. 